STRUCTURAL SCIENCE CRYSTAL ENGINEERING MATERIALS

ISSN 2052-5206

Keywords: book review; inorganic crystals.

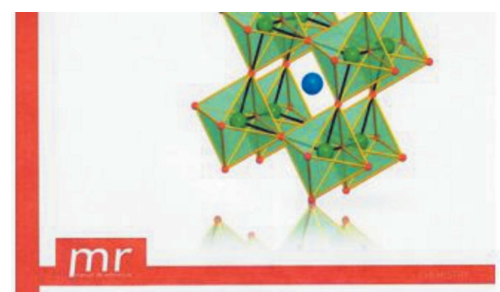

STRUCTURAL MODELS OF INORGANIC CRYSTALS FROM THE ELEMENTS TO THE COMPOUNDS Angel Vegas

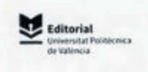

C 2020 International Union of Crystallography

\section{Structural Models of Inorganic Crystals. From the Elements to the Compounds. By Ángel Vegas. Editorial Universitat Politècnica de València. 2018. Hardback, Pp. xxviii+444. Price Euro 99. ISBN 9788490486023}

\author{
Sergey V. Krivovichev*
}

NanoMaterials Research Centre, Kola Science Centre, Russian Academy of Sciences, Apatity, Russian Federation, and Department of Crystallography, St. Petersburg State University, St. Petersburg, Russian Federation. *Correspondence e-mail: s.krivovichev@ksc.ru

Despite the exponential explosion of the number of research papers dealing with organic and metal-organic compounds (e.g. metal-organic frameworks, polymorphism of organic crystals, crystal engineering, etc.), inorganic crystalline materials continue to attract attention from material scientists, crystallographers, condensed matter physicists, solid state chemists and mineralogists. However, there have been very few books published so far in the XXI century that provide an overview of structural correlations existing between various classes of inorganic compounds based upon the analysis of their structural architecture. The book by Ángel Vegas is a comprehensive (yet provocative and quite original, see below) treatise on crystal chemistry, where the author unifies a large amount of data to construct a coherent conceptual framework for understanding the large family of inorganic structures usually interpreted through the ionic model. The very basic question the author asks is 'Why do atoms occupy the positions they have in the structures?', which is given as the title of the Chapter 16. Which factors govern the arrangement of ions in ionic crystals? What is the best way to describe the structure of an inorganic crystal so that correlations to related compounds are immediately seen and understood? The subtitle of the book provides a partial answer: 'from the elements to the compounds'. In general, the idea that structures of many inorganic oxides, halogenides and chalcogenides can be described as derivatives of parent metals or intermetallides is not new (and the author provides detailed historical notes here and there). However, Vegas wants to demonstrate how these analogies arise from the nature of interatomic interactions in ionic crystals, gathering evidence from different fields of modern research in condensed matter physics, materials and solid state chemistry.

The author develops his approach point-by-point in a manner quite accessible to the reader with a primary knowledge of crystallography and inorganic chemistry. The book contains 18 chapters, which are subdivided into 151 subchapters, each with an average length of three book pages. The first three chapters are devoted to the description of the basic concept of crystal chemistry of the compounds that are usually called 'ionic'. Vegas sharpens his analysis against the 'ionic model' that ' ... describes the structures as closestpacked arrays of bulky anions with small cations occupying interstices of that anionic array' (p. 13). Chapter 4 points out the necessity of alternative approaches, providing some historical examples of structural and geometrical correlations between the cation arrays in the structures of oxides and their parent metals and alloys. This theme is continued in Chapters 6 and 7, where the author comes to the conclusion that 'oxides are real oxygen-stuffed alloys (my italics) whenever we take into account the high-pressures phases' (p. 143). We note the rather strange title for subchapter 7.6 'Cations are not isolated entities...', a somewhat incorrect statement, taking into account the range of interactions that cations in oxides are involved in. However, before this conclusion Chapter 5 introduces Zintl phases and the Zintl-Klemm concept with the idea of electron delocalization between metal atoms (e.g. the $\left[\mathrm{Al}_{2}\right]^{2-}$ subarray in $\left.\mathrm{SrAl}_{2}\right)$. The author wants us to start to believe that cation-cation interactions in oxides have something in common with metal-metal interactions in Zintl phases. The idea is further promoted in Chapters 8 to 10 that continue to add examples of similarities (or even geometrical identities) of cation arrays in oxides and elemental structures. Therefore, the reader is not surprised 
that the title of Chapter 11 is 'Why the elemental structures are preserved in their oxides?' The answer given at the end of this short chapter is: '.. valence electrons can separate in the solids in the form of non-nuclear maxima (NNM) that are located at the same position occupied by the $\mathrm{O} / \mathrm{S}$ atoms in the corresponding oxides/sulfides' (p. 229). In my opinion, this is a major insight that may explain the whole range of observed crystal-chemical correlations between dense oxides and their parent metals/alloys. The author, however, does not develop this useful point immediately, but concentrates on what he calls 'the extended Zintl-Klemm concept', which is considered in Chapters 12 to 14, first for aluminates (Chapter 12) and then for silicates (Chapters 13 and 14). In order to understand the approach undertaken by the author, imagine that all zeolite frameworks described as three-dimensional nets formed by $\mathrm{T}$ atoms (e.g. $\mathrm{Al}$ and $\mathrm{Si}$ in aluminosilicate zeolites) are based in fact upon $\mathrm{T}-\mathrm{T}$ (and not $\mathrm{T}-\mathrm{O}-\mathrm{T}$ !) bonding interactions! The second-neighbour interactions are certainly of importance, but can hardly be considered as attractive bonding. Chapter 16 generalizes the extended Zintl-Klemm concept, whereas Chapters 15 and 18 discuss its various electronic aspects, where again the important insight (localized electron clouds as 'pseudo-atoms') outlined in Chapter 11 appears. Chapter 17 explains that the concept developed by the author allows for an easier comparison between different structure types and a better understanding of the organization of the crystal structure.

Despite (or maybe due to) its provocative character and unusual style, the book is an extremely useful and enjoyable read. The reader may disagree with the author, but his thoughts and ideas certainly deserve some meditation and analysis. Some shortcomings, however, should be pointed out. In particular, the reader may get an impression that the author pretends that the developed model is more universal than it actually is. Perhaps, this is an aberration that can be derived from some inaccurate expressions. For instance, the author points out that '.. in the majority (sic - S.V.K.) of the structures, the topology of the cation array corresponded either to elemental structures or to simple alloys' (p. 54). It can easily be shown that there are many complex structures (particularly, in oxides and oxysalts) that cannot be treated as stuffed cation arrays derived from parent metals or alloys. The approach is more applicable to 'dense' oxides with rather simple stoichiometries than to open structures with complex chemical compositions. There are some structural misinterpretations [e.g. the feldspar and coesite topologies are different (p. 302), despite the fact that both contain fourmembered tetrahedral rings]. The book also contains some small and rather unfortunate problems with the standard crystallographic nomenclature: the space-group symbols are written with the lattice symbols in bold (e.g. Pnma instead of Pnma and $\boldsymbol{P} 2_{1} 2_{1} 2_{1}$ instead of $P 2_{1} 2_{1} 2_{1}$ ), against the current IUCr rules, structure diagrams have no identification of crystallographic directions (which, however, makes them less cumbersome).

In general, the book provides an interesting and useful review of inorganic structures derived from the structures of elements and intermetallics with the introduction into modern concepts of chemical bonding in inorganic crystals. It is recommended to any reader interested in crystallography, inorganic chemistry, mineralogy and related disciplines. It cannot be considered as a textbook on inorganic crystal chemistry (and it was not written for this purpose!), but can nevertheless be used in teaching crystallography and structural chemistry. The quality of printing, on excellent paper and with many coloured structure diagrams, is very high. The book is pleasant to keep at hand and read during coffee hour or afternoon tea (whichever one prefers). 